\title{
Left sinus of Valsalva aneurysm presenting as myocardial ischemia
} \author{
Baltimore, Md
}

Malini Sarkar, ${ }^{\mathrm{a}}$ Brody Wehman, MD, ${ }^{\mathrm{a}}$ Ratnakar Mukherjee, MD, ${ }^{\mathrm{b}}$ and Bradley S. Taylor, MD, MPH, ${ }^{\mathrm{a}}$

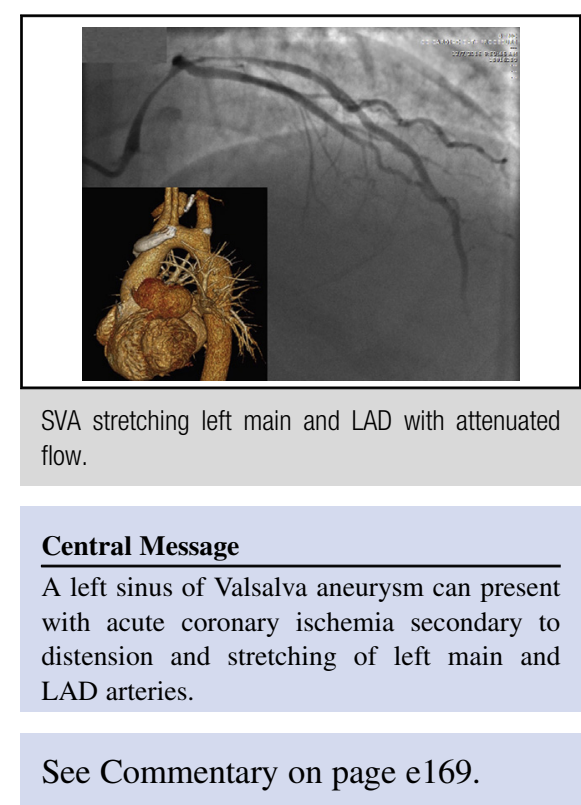

postoperative course was notable for re-exploration for cardiac tamponade, atrial fibrillation, and temporary renal insufficiency. The patient made a full recovery and was asymptomatic at follow-up 4 months later and is alive 2 years postoperatively (Figure 2).

\section{DISCUSSION}

Most SVAs arise from the right sinus (98\%) and are clinically silent lesions. This unusual left SVA elongated and attenuated the left main coronary artery, causing coronary ischemia and exertional chest pain.

The patient was being followed for a history of coarctation. At presentation, the coarctation was asymptomatic and the patient had no clinical sequlae seen in patients with significant coarctation of the aorta. We also assessed the coarctation using computed tomography angiography and in the operating room. The diameter reduction was not considered to be significant and the gradient was only 12 to $14 \mathrm{~mm} \mathrm{Hg}$ across the coarctation.

Coronary artery bypass grafting was performed because of the uncertain response of the left main coronary artery and proximal left anterior descending to relief of the extrinsic distortion. The vessel was significantly stretched, narrow, and attenuated. Because of the chronic nature of this process, we could not predict that relief of the stretch on the vessel would restore normal flow in this 


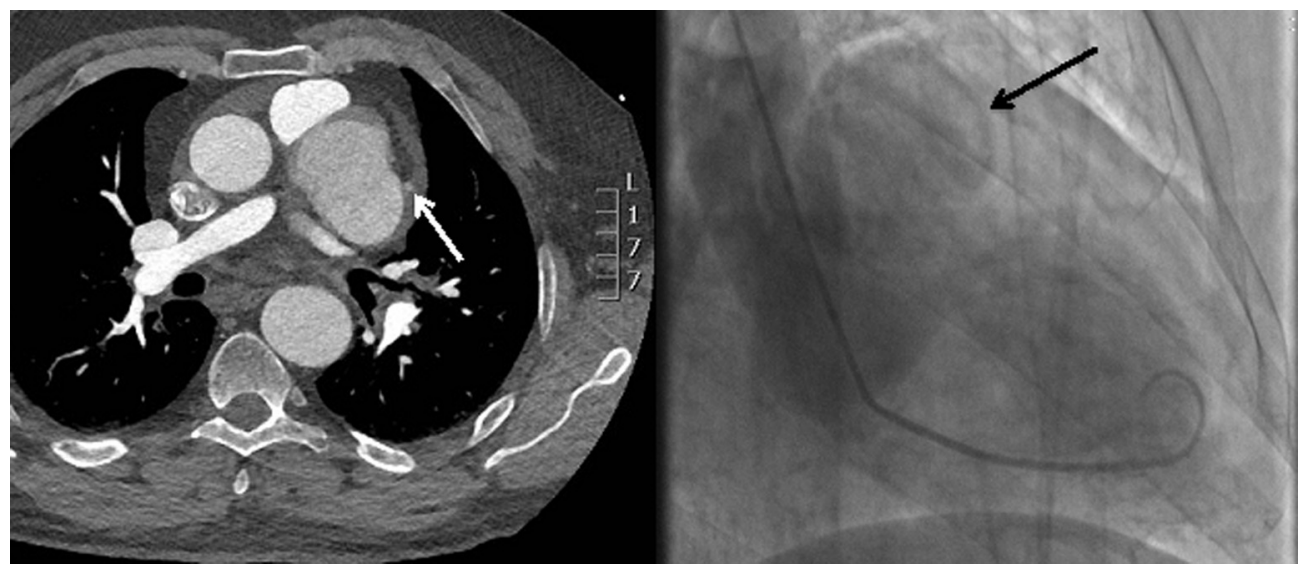

FIGURE 1. Computed tomography scan (left) of large left sinus of Valsalva aneurysm with distortion of the left anterior descending artery (arrow). Ventriculogram (right) showing delayed filling of the sinus of Valsalva aneurysm (arrow).

critical vessel (Central Image). Treatment of left SVA has included aortic root repair, aortic valve replacement, coronary artery bypass, and aortic valve repair. ${ }^{1}$ An alternate

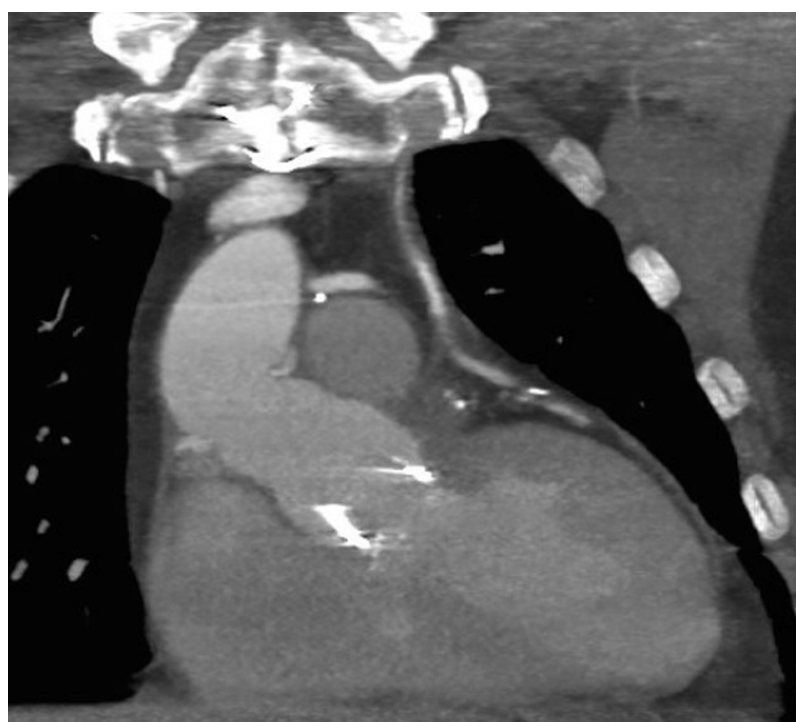

FIGURE 2. Postoperative computed tomography scan showing repair of sinus of Valsalva aneurysm and left internal thoracic artery to the left anterior descending artery graft. mechanism for myocardial ischemia from SVA has been described, in which aortic regurgitation causes myocardial ischemia and infarction. ${ }^{1}$ Most left SVAs are asymptomatic and might present as sudden cardiac death, or they present with severe aortic insufficiency. ${ }^{2}$ Takahara and colleagues describe the case of an isolated aneurysm of the left sinus of Valsalva presenting as aortic valve regurgitation ${ }^{3}$ whereas others describe rare cases of left SVA causing myocardial ischemia. ${ }^{4,5} \mathrm{We}$ are the first, to our knowledge, to report distortion of the coronary as the cause for ischemia.

\section{References}

1. Sasson L, Katz MG, Ezri T, Tamir A, Rozenman Y, Geva Y, et al. Ruptured sinus of Valsalva aneurysm complicated by myocardial ischemia: pathogenetic mechanisms. Cardiovasc Pathol. 2006;15:291-3.

2. Martínez-Comendador J, Gualis J, Martín CE, Santamaria A. Left main coronary artery compression by a left sinus of Valsalva aneurysm. Interact Cardiovasc Thorac Surg. 2013;16:713-4.

3. Takahara Y, Sudo Y, Sunazawa T, Nakajima N. Aneurysm of the left sinus of Valsalva producing aortic valve regurgitation and myocardial ischemia. Ann Thorac Surg. 1998;65:535-7.

4. Cuculi F, Rossi M, Bradley KM, Westaby S. Rupture of a left sinus of Valsalva aneurysm with coronary compression: a rare cause of ischemic chest pain. Ann Thorac Surg. 2011;92:e97-9.

5. Bashour TT, Chen F, Yap A, Mason DT, Baladi N. Fatal myocardial ischemia caused by compression of the left coronary system by a large left sinus of Valsalva aneurysm. Am Heart J. 1996;132:1050-2. 